Revista Saúde.Com

Rev. Saúde.Com 2020; 16(2): 1852-1858

ISSN 1809-0761

www.uesb.br/revista/rsc/ojs

\title{
SEMÁFORO DO CORAÇÃO E BINGO CARDIOVASCULAR: TECNOLOGIAS EDUCATIVAS PARA PREVENÇÃO DE DOENÇAS CARDIOVASCULARES EM IDOSOS
}

\author{
HEART SEAMPHOR AND BINGO CARDIOVASCULAR: EDUCATIONAL \\ TECHNOLOGIES TO PREVENT CARDIOVASCLAR DISEASES IN ELDERLY
}

Luciane Silva Oliveira, Kairo Cardoso da Frota, Yandra Kelline Brandão Braga, Maria
Aparecida Fernandes Cardoso, Keila Maria de Azevedo Ponte

Universidade Estadual Vale do Acaraú

\begin{abstract}
The aim of this paper is to describe the use of educational technologies to prevent cardiovascular disease in the elderly: Heart Semaphore and Cardiovascular Bingo. This is an experience report, conducted in November 2018, with a group of elderly people from a Basic Health Unit in the city of Sobral-Ceará. Health education was developed through a practical workshop. Fifteen people from the group participated. The moment was organized in the following phases: group integration and presentation; research on their knowledge of heart disease through the "Heart Traffic Light" technology and guidance on habits for the prevention of cardiovascular disease through the "Bingo Cardiovascular" technology. It was evidenced that the use of educational technologies favored the process of communication between facilitators and participants, because it is possible to work the theme in a playful and creative way, without the need of impositions, providing the resignification of the learning process. The elaboration and implementation of the educational technologies were relevant for instigating the creativity of the facilitators and facilitating the sharing of knowledge.
\end{abstract}

Key words: Cardiovascular diseases; Health Promotion; Education nursing.

\section{Resumo}

O objetivo do presente artigo é descrever a utilização das tecnologias educativas para prevenção do adoecimento cardiovascular em idosos: Semáforo do Coração e Bingo Cardiovascular. Trata-se de um relato de experiência, realizado em novembro de 2018, com um grupo de idosos de uma Unidade Básica de Saúde do município de Sobral-Ceará. A educação em saúde foi desenvolvida através de uma oficina prática. Participaram 15 pessoas integrantes do grupo. O momento foi organizado nas seguintes fases: integração com o grupo $e$ apresentação; investigação acerca de seus conhecimentos sobre cardiopatias através da tecnologia "Semáforo do Coração" e orientações acerca dos hábitos para a prevenção de doenças cardiovasculares através da tecnologia "Bingo Cardiovascular". Evidenciou-se que o uso das tecnologias educativas favoreceu o processo de comunicação entre facilitadores e participantes, pois pode-se trabalhar a temática de forma lúdica e criativa, sem a necessidade de imposições, proporcionando a ressignificação do processo de aprendizagem. A elaboração $e$ implementação das tecnologias educativas foram relevantes por instigar a criatividade dos facilitadores e facilitar o compartilhamento de conhecimentos.

Palavras-chave: Doenças cardiovasculares; Promoção da saúde; Educação em enfermagem. 


\section{Introdução}

As Doenças Cardiovasculares (DCV) ainda prevalecem como a principal causa de morte e incapacidade no Brasil e no mundo. De acordo com a Organização Mundial da Saúde (OMS), em 2008, 17,3 milhões de pessoas foram a óbito em decorrência desse grupo de enfermidades ${ }^{1}$.

Além da morbimortalidade, também geram um elevado custo ao sistema de saúde, com aumento significativo nos últimos anos. Considerando a relevância impactante em termos de saúde e gastos públicos, medidas preventivas devem ser tomadas para além do tratamento precoce e correto, focando-se em controlar os fatores de riscos, como obesidade, sedentarismo e tabagismo ${ }^{2}$.

Nesse contexto, a prevenção de doenças e a promoção da saúde nunca foram tão urgentes. A necessidade de promover a saúde da população e, consequentemente, de diminuir os índices de morbidade e mortalidade por doenças crônicas, dentre elas as DCV, tem levado a reflexões sobre a utilização de estratégias de promoção da saúde eficientes ${ }^{3}$.

Entre essas ações destacam-se aquelas que visam o aumento do conhecimento da população sobre fatores de risco para as doenças crônicas, uma vez que gera o empoderamento para mudanças no estilo de vida e, consequentemente, pode ser útil para melhorar a qualidade de vida, evitar o surgimento de um agravo e influenciar na busca pelo tratamento quando a doença já está estabelecida ${ }^{1}$.

A Educação em Saúde é vista como um meio para alcançar e disseminar maior conhecimento para compreensão da população acerca do seu estado de saúde. Dessa forma, fazse necessária a ampliação dos investimentos que envolvam essa temática em saúde, a fim de que possam ser atingidos os objetivos relacionados aos cuidados em saúde ${ }^{4}$.

Intervenções educacionais realizadas em ambiente de atenção primária focadas em modificação de hábitos de vida mostram-se eficazes na redução do risco cardiovascular e controle dos fatores de riscos. Além disso, podemos aproveitar o contato com outros níveis de saúde para realizar intervenções ${ }^{2}$.

Logo, o uso de determinadas tecnologias, inclusive aquelas que contam com o auxílio da rede mundial de computadores, amplia a abordagem e dá nova característica para as práticas educativas de forma inovadora, capaz de envolver grupos específicos, a exemplo dos idosos nestas ações 4 .

Assim, a tecnologia educacional tem se destacado por proporcionar educação e promoção da saúde à população ao permitir a identificação sistemática de desenvolvimento, organização ou utilização de recursos educacionais e manuseio desses processos, bem como o uso das técnicas orientadas por equipamentos ou auxílio de recurso audiovisual no cenário educacional ${ }^{3}$.

Portanto, a estratégia educativa utilizadas trazem impactos diretos na saúde dos idosos, e por isso o uso de tecnologias educativas nesse público vulnerável torna-se uma tendência de estudo e realizações para ações de promoção à saúde ${ }^{5}$.

Com base no exposto, pretende-se elucidar experiências exitosas do Grupo de Pesquisa em Vulnerabilidade em Saúde (GEVS) da Universidade Estadual Vale do Acaraú (UVA), no qual há o desenvolvimento e criação de tecnologias educativas para a promoção da saúde cardiovascular. Assim, o objetivo desse estudo é descrever a utilização das tecnologias educativas "Semáforo do Coração" e "Bingo Cardiovascular" para a prevenção do adoecimento cardiovascular em idosos.

\section{Metodologia}

Trata-se de um relato de experiência, realizado em novembro de 2018 em uma Unidade Básica de Saúde (UBS), no município de Sobral-Ceará, com um grupo de idosas representado por quinze participantes.

Antes de planejar a intervenção educativa, os pesquisadores realizaram uma busca na literatura cientifica, de modo a compreender melhor a temática e analisar as publicações relacionadas à educação em saúde e ao uso de tecnologias educativas com idosos. Posteriormente, foi consultado qual melhor tecnologia para ser utilizada com o público-alvo.

Assim, foram utilizadas duas tecnologias educativas: Semáforo do Coração e Bingo Cardiovascular, as quais foram construídas pelos autores do presente estudo. A aplicação foi realizada em um único encontro de extensão com duração de três horas, onde as atividades 
desenvolvidas foram planejadas e executadas por acadêmicas de enfermagem e participantes do Grupo de Estudos em Vulnerabilidade e Saúde (GEVS) da área temática Saúde Cardiovascular.

A intervenção educativa foi desenvolvida em três etapas: a primeira foi um momento de integração com o grupo e apresentação; a segunda foi uma breve investigação acerca de seus conhecimentos sobre DCV através da tecnologia "Semáforo do Coração" e a terceira foi a realização de orientações sobre os hábitos para a prevenção DCV através da tecnologia "Bingo Cardiovascular". No quadro 1 apresenta-se o planejamento da intervenção educativa.

Figura 1. Planejamento de intervenção educativa com um grupo de idosas sobre DCV.

\section{Integração com o} grupo Conhecer o perfil das participantes

- Foram realizadas perguntas através de sorteio para descobrir o perfil das participantes a nível de conhecimento sobre as DCV e saber quais as principais doenças crônicas prevalentes.

\section{Semáforo do coração}

Investigar qual o nivel de conhecimento sobre DCV de cada participante do grupo.

-Foram entregues figuras ilustrativas que podem aumentar as chances das DCV e outras figuras com maneiras que diminuem as chances das DCV. Cada particpante colocava a sua imagem em uma cartolina de cor vermelho, amarelo ou verde.

Bingo Cardiovascular Conscientizar o grupo sobre quais os hábitos devem ser adquiridos para prevenção DCV.

- Cada participante ganhou uma cartela, com 12 imagens ilustrativas diferentes, e o administrador do jogo fazia um sorteio para uma pergunta e a resposta era correspondente a uma imagem que poderia estar ou não presente na cartela. Cada imagem sorteada iria sendo justificado qual os alimentos devem ser mais ou menos consumidos para prevenção do DCV.

\section{Relato de experiência}

O grupo de idosos era composto apenas por pessoas do gênero feminino, o qual totalizava 15 participantes. A idade variou de 60 a 70 anos. Todas possuíam pelo menos uma doença crônica não transmissível, sendo que $60 \%$ (9) possuíam hipertensão, diabetes mellitus e dislipidemia simultaneamente, $20 \%$ (3) eram cardiopatas e $20 \%$ (3) eram apenas diabéticas. Quanto à escolaridade, dez possuíam ensino fundamental completo, e cinco eram analfabetas. Todas residem próximo à UBS e afirmaram frequentá-lo frequentemente.
O momento da Intervenção Educativa foi divido em etapas principais, as quais, terão seus resultados descritos separadamente.

Integração Com $O$ Grupo

De início foram distribuídas quinze perguntas aleatórias entre as participantes para se discutir os hábitos de vida mais comuns entre as mesmas e conversar acerca das doenças que as acometiam.

Entre as perguntas tinham: "Você faz atividade física? Se sim, quantas vezes por semana?", "Você costuma comer frituras, quantas vezes por semana?", "Quais as frutas que você consome durante o dia?", "Você tem o 
hábito de tomar suas medicações no horário correto?", entre outros questionamentos, todos voltados para promover o conhecimento acerca do grupo em questão.

Cada participante respondia
prioritariamente a uma pergunta, mas todas eram convidadas a comentar 0 aspecto perguntado, com o objetivo de proporcionar uma discussão mais ampla entre todos os componentes do grupo.

Breve investigação do conhecimento acerca do adoecimento cardiovascular: semáforo do coração

O segundo momento foi desenvolvido tendo como subsídio a tecnologia educativa "Semáforo do Coração". Tratou-se de uma avaliação acerca do conhecimento das participantes quanto ao adoecimento cardiovascular. Esta avaliação ocorreu por meio da entrega aleatória às participantes de figuras ilustrativas de aspectos que aumentam a chance de desenvolvimento de DCV e/ou de ilustrações que representavam hábitos de prevenção de cardiopatias. As participantes eram orientadas a colocar as imagens em cartolinas de cores vermelha, amarela ou verde, simulando um semáforo, de acordo com 0 que elas consideravam que a figura era. A cor vermelha era destinada à colagem de imagens que representam hábitos não saudáveis à saúde do coração, a cor amarela à hábitos considerados saudáveis quando utilizados em poucas proporções e de cor verde os hábitos saudáveis. Nenhum dos facilitadores intervia neste momento pois foi destinado para compreender o nível de conhecimento do grupo.

A partir da aplicação da tecnologia, pode-se discutir diversos aspectos que circundem o cuidado à saúde do coração. Pode-se compreender que dentre as quinze participantes, nove alegaram não ter acompanhamento com especialista pois apenas fazem a retenção de medicamentos na farmácia da UBS, realizando a renovação a cada seis meses, sem realização de consulta qualificada; três referiram fazer o uso de marca-passo e ter o acompanhamento anualmente com o cardiologista e as demais relataram buscar atendimento em clínica particular em casos de queixas prevalentes, mas não mantinham esse atendimento como rotina.
Ao decorrer do desenvolvimento do Semáforo do Coração observou-se que, quanto ao autocuidado, a maioria das participantes alegraram tentar manter sempre hábitos saudáveis, como fazer caminhadas todos dias, alimentar-se de uma alimentação balanceada e tomar a medicação na hora certa, apenas algumas participantes declararam ter cuidado com a alimentação apenas quando apresentava alguma queixa.

Em relação aos principais desafios quanto ao autocuidado, emergiram as seguintes fragilidades: dificuldade em ter atendimento pela Unidade Básica de Saúde, falta de recursos financeiros para buscar um atendimento particular e falta de momentos com profissionais para melhores esclarecimentos sobre quais os hábitos saudáveis devem ser realizados.

A descrição das imagens selecionadas para cada uma das cartolinas ficou a cargo das participantes. As mesmas foram convidadas a explanar as suas escolhas, apesar de algumas apresentarem convicções errôneas. Algumas participantes pensavam que era correto o uso excessivo de óleo de girassol, ou de coco, como também o uso de excessivo de margarida com baixo teor de gordura, essas justificativas foram com as figuras da cartolina verde, as quais foram corrigidas e orientadas corretamente.

Quanto as imagens da cartolina amarela, as participantes destacaram o consumo de cereais, como biscoitos, pães e bolos. Muitas afirmaram que esses alimentos poderiam ser consumidos até três vezes por semana, enquanto os carboidratos integrais poderiam ser ingeridos todos os dias, sem limites de quantidade.

A última cartolina exposta foi a vermelha, na qual as imagens estavam totalmente corretas, sendo expressos os alimentos que menos devem ser consumidos, como carne de origem do boi ou porco, guloseimas, frituras e alimentos industrializados, além de alimentos ricos em sódio e temperos industriais.

\section{Orientações sobre hábitos saudáveis à saúde cardiovascular: bingo cardiovascular}

Por fim, com a finalidade de realizar orientações acerca dos hábitos saudáveis ao coração, aplicou-se o Bingo Cardiovascular, o qual trata-se de um jogo educativo composto por cartas de bingo que contém ilustrações, em vez 
de números, as quais são respostas de perguntas que são sorteadas pelo facilitador. As cartas foram distribuídas de forma aleatória e, de acordo com a pergunta sorteada, o participante assinalava a imagem como resposta, até o preenchimento total da cartela. A cada pergunta sorteada era realizado um momento de reflexão sobre a imagem para esclarecimento de dúvidas.

Como se tratou da última atividade do encontro, cada participante foi convidado a expressar seus sentimentos diante da intervenção. Foi perceptível, assim, a adesão das participantes e o entusiasmo em aplicar os conhecimentos aprendidos.

\section{Resultados e Discussão}

Diante da magnitude do desenvolvimento das DCV no momento histórico atual, várias são as estratégias possíveis de se trabalhar a prevenção. Em pesquisa ${ }^{6}$, afirma-se que a prevenção através de mudanças no estilo de vida e no ambiente da população revela-se como uma forma mais eficaz de controlar a epidemia de DCV, sendo a educação em saúde, por meio de tecnologias educativas, uma estratégia relevante.

Durante a realização de educação em saúde com a população faz-se necessário primeiramente a comunicação efetiva, através do estabelecimento de vínculos com o grupo. É importante que nessa fase, os facilitadores busquem conhecer o histórico de cada cliente que deseja participar, para assim proporcionar uma intervenção eficaz com troca de saberes.

A educação em saúde, embora possua métodos e segmentos distintos, não se limita apenas a transmitir conhecimento, mas estabelece vínculos entre assistidos e profissionais, e promove a participação ativa do paciente e constantes remodelagens conceituais destes indivíduos quanto a hábitos que comprometam a saúde e a qualidade de vida ${ }^{7}$.

Desta forma, percebe-se os benefícios estabelecidos entre a educação em saúde utilizando-se de tecnologias leves para o auxílio dos clientes e facilidade de incorporar informações das mais complexas à vida da pessoa. Tratando-se de doenças cardiovasculares, é notável, principalmente em regiões mais carentes, que a população pouco sabe sobre estas.
Assim como mostram os estudos realizados em outros países que revelam que o conhecimento sobre os fatores de risco para DCV é escasso, e que as questões socioeconômicas (escolaridade e renda em particular) estão associadas ao baixo entendimento dos mesmos. No Brasil, os estudos realizados, a maioria de base populacional, confirmam os achados dos outros países ${ }^{8}$.

Com base no exposto, notou-se que a integração da população para o cuidado com as doenças cardiovasculares tem demonstrado os bons resultados, e a utilização de métodos ativos podem despertar sobre a importância das doenças. A aproximação entre o setor da saúde com o da educação pode fomentar, nos territórios, a compreensão da saúde como um processo socialmente construído ${ }^{9,10}$.

Categorizar o conhecimento das participantes foi uma forma de conhecer a visão das idosas sobre os problemas que afetavam diretamente suas vidas e, assim poder intervir diretamente com soluções e dicas para melhorar o auto-cuidado e o enfretamento de doenças. É importante deixar que o público se sinta à vontade para questionar suas dúvidas e também colaborar com conhecimentos específicos.

O conhecimento sobre um determinado desfecho em saúde pode ser útil para ajudar a evitar o surgimento de um agravo, podendo também influenciar na busca pelo tratamento, quando a doença já está estabelecida. Para tanto, espaços de uso e fluxo populacional, como escolas, universidades, veículos de comunicação e serviços de saúde, são potenciais difusores dessa informação, ainda que a forma de impactar perante a população geral possa ser distinta².

Assim, na prática da educação em saúde, a tecnologia deve ser utilizada de modo a favorecer a participação dos sujeitos no processo educativo, contribuindo para a construção da cidadania e o aumento da autonomia dos envolvidos. Tanto na educação quanto na saúde, os educadores devem compreender as tecnologias como meios facilitadores dos processos de construção do conhecimento, numa perspectiva criativa, transformadora e crítica ${ }^{11}$.

O uso de dinâmica em grupo de portadores de doenças crônicas pode ser usada como estratégia de empoderamento pois os participantes participam, refletem, aprendem 
com a experiência do outro, compartilha ideias e estimula o raciocínio além, do respeito mútuo ${ }^{12}$.

\section{Conclusão}

Percebeu-se, a partir do desenvolvimento do presente estudo, que o grupo de idosos em análise possui conhecimento eficiente acerca do processo de adoecimento cardiovascular e das estratégias de prevenção. Evidenciou-se que o uso das tecnologias educativas favoreceram o processo de comunicação entre facilitadores e participantes, pois pode-se trabalhar a temática de forma lúdica e criativa, sem a necessidade de imposições, proporcionando a ressignificação do processo de aprendizagem.

Foi notório, ainda, a motivação do público alvo para a participação no momento, visto que foi evidenciado que não se tratava de um espaço para punições de elucidações de práticas não saudáveis mas sim de promoção da conscientização para o autocuidado.

A principal fragilidade evidenciada foi a da aplicação das tecnologias educativas em apenas um encontro, contudo, tratou-se de um momento esclarecedor e de tira-dúvidas, pois sugere-se que essas tecnologias devem ser realmente utilizadas uma única vez em cada grupo, para evitar repetições, o que potencialmente pode gerar desinteresse nos participantes envolvidos.

Por fim, a elaboração e implementação das tecnologias educativas foram relevantes por instigar a criatividade dos acadêmicos de enfermagem ao desenvolver novos conhecimentos de estratégias para prevenção do adoecimento cardiovascular. Sugere-se a replicação dessa estratégia em todos os âmbitos do sistema de saúde, como forma de tornar mais didática a discussão acerca de um assunto tão sério e relevante à saúde de todos.

\section{Referências}

1. Ganassin GS, et al. Efetividade da intervenção educativa no conhecimento de homens relacionado às doenças cardiovasculares. Acta Paulista de Enfermagem [internet]. 2016 Jan [acesso em 15 jul 2019]; 29 (1):38-46, Janeiro, $2016 . \quad$ Disponível em: http://www.scielo.br/pdf/ape/v29n1/1982-0194ape-29-01-0038.pdf.
2. Neto AS, et al. Importância da Educação para prevenção cardiovascular. Revista da Sociedade de Cardiologia do Estado de São Paulo [internet]. 2019 Mar [acesso em 20 ago 2019]; 20(1):43-45. Disponível em: http://socesp.org.br/revista/edicaoatual/importancia-da-educacao-para-prevencaocardiovascular/103/681/.

3. Souza ACC, Moreira TMM, Borges JWP. Tecnologias educacionais desenvolvidas para promoção da saúde cardiovascular em adultos: revisão integrativa. Revista Escola de Enfermagem USP [internet]. 2014 Out [acesso em 15 ago 2019]; 48(5):944-951. Disponível em: <http://www.scielo.br/scielo.php?script=sci_artt ext\&pid=S0080-

62342014000500944\&lng=en\&nrm=iso>.

4. Silva CTS, Carvalho JM, Carvalho FLQ. Tecnologias Voltadas para Educação em Saúde: O que temos para a saúde dos idosos. II STAES: Seminário de Tecnologias Aplicadas a Educação em Saúde. Revista UNEB [internet]. 2015 Out [acesso em 15 ago 2019]. Disponível em: <https://www.revistas.uneb.br/index.php/staes/ article/view/1615>.

5. Frota $\mathrm{KC}$, et al. Tecnologias educativas: estratégias eficientes para a promoção da saúde de idosos. Revista Saúde.Com [internet]. 2019 Ago [acesso em 10 ago 2019]; 15(2):1531-1537. Disponível

em: http://periodicos2.uesb.br/index.php/rsc/article/ view/4401/4094.

6. Tomiak E, et al. Prevention of cardiovascular disease in a rural general practice. Ann Agric Environ Med [internet]. 2016 [acesso em 20 ago 2019]; 23(4):553-558. Disponível em: https://www.ncbi.nlm.nih.gov/labs/articles/ 28030922/.

7. Janini JP, Bessler D, Vargas AB. Educação em saúde e promoção da saúde: impacto na qualidade de vida do idoso. Saúde Debate [internet]. 2015 Abr [acesso em 18 jul 2019]; 39(105):480-490. Disponível em: <http://www.scielo.br/scielo.php?script=sci_artt ext\&pid=S0103-

$11042015000200480 \&|n g=e n \& n r m=i s o \& t| n g=p t>$ 8. Bonotto GM, Mendoza-sassi RA; Susin LRO. Conhecimento dos fatores de risco modificáveis para doença cardiovascular entre mulheres e seus fatores associados: um estudo de base populacional. Ciência \& Saúde Coletiva [internet]. 2016 [acesso em 15 ago 2019]; 1(21): 
293-302. Disponível em:

http://www.scielo.br/pdf/csc/v21n1/1413-8123-

csc-21-01-0293.pdf.

9. Santos NF, et al. Combate a dengue nas escolas: formando multiplicadores para vencer essa batalha. Suplemento Revista Interface Comunicação, Saúde, Botucatu, supl. 3, 2014. Disponível em: <http://conferencias.redeunida.org.br/docs/inde x.php/redeunida/RU11/paper/view/3049>.

10. BRASIL. Ministério Educação. Secretaria de Educação Profissional e Tecnológica. Catálogo nacional de cursos técnicos e tecnológicos/agentes de vigilância em saúde. 3. ed. Brasília; 2016.

11. Borges TT, et al. Conhecimento sobre fatores de risco para doenças crônicas: estudo de base populacional. Cad. Saúde Pública [internet]. 2014 Jul [acesso em 16 jul 2019]; 7(25):1511$1520 . \quad$ Disponível em: http://www.scielo.br/pdf/csp/v25n7/09.pdf.

12. Martins AKL, et al. Literatura de cordel: tecnologia de educação para saúde e enfermagem. Revista de Enfermagem UERJ [internet]. 2011 [acesso em 20 ago 2019]; 19(2):324-329. Disponível em: http://www.facenf.uerj.br/v19n2/v19n2a25.pdf .

\section{Endereço para Correspondência}

Universidade Estadual Vale do Acaraú

Av. Transnordestina, s/n, Novo Horizonte - BA, 44036-900

e-mail: lusoliveira2014@gmail.com

Recebido em 13/10/2019

Aprovado em 15/12/2020

Publicado em 19/01/2021 